Scientific Paper

\title{
Using of Laplacian Re-decomposition image fusion algorithm for glioma grading with SWI, ADC, and FLAIR images
}

\author{
Amir KHORASANII, Mohamad Bagher TAVAKOLI ${ }^{\mathrm{a},{ }^{*},}$, Masih SABOORI ${ }^{\mathrm{b}}$ \\ ${ }^{a}$ Department of Medical Physics, School of Medicine, Isfahan University of Medical Sciences, Isfahan, Iran \\ ${ }^{b}$ Department of Neurosurgery, School of Medicine, Isfahan University of Medical Sciences, Isfahan, Iran \\ ${ }^{*}$ E-mail address: mbtavakoli@mui.ac.ir
}

\begin{abstract}
Introduction: Based on the tumor's growth potential and aggressiveness, glioma is most often classified into low or highgrade groups. Traditionally, tissue sampling is used to determine the glioma grade. The aim of this study is to evaluate the efficiency of the Laplacian Re-decomposition (LRD) medical image fusion algorithm for glioma grading by advanced magnetic resonance imaging (MRI) images and introduce the best image combination for glioma grading.

Material and methods: Sixty-one patients (17 low-grade and 44 high-grade) underwent Susceptibility-weighted image (SWI), apparent diffusion coefficient (ADC) map, and Fluid attenuated inversion recovery (FLAIR) MRI imaging. To fuse different MRI image, LRD medical image fusion algorithm was used. To evaluate the effectiveness of LRD in the classification of glioma grade, we compared the parameters of the receiver operating characteristic curve (ROC).

Results: The average Relative Signal Contrast (RSC) of SWI and ADC maps in high-grade glioma are significantly lower than RSCs in low-grade glioma. No significant difference was detected between low and high-grade glioma on FLAIR images. In our study, the area under the curve (AUC) for low and high-grade glioma differentiation on SWI and ADC maps were calculated at 0.871 and 0.833 , respectively.

Conclusions: By fusing SWI and ADC map with LRD medical image fusion algorithm, we can increase AUC for low and high-grade glioma separation to 0.978. Our work has led us to conclude that, by fusing SWI and ADC map with LRD medical image fusion algorithm, we reach the highest diagnostic accuracy for low and high-grade glioma differentiation and we can use LRD medical fusion algorithm for glioma grading.
\end{abstract}

Key words: glioma; laplacian re-decomposition; susceptibility-weighted imaging; diffusion-weighted imaging; image fusion.

\section{Introduction}

Glioma represents roughly 30 percent of all brain tumors and central nervous system tumors and 80 percent of all malignant brain tumors. ${ }^{1}$ Glioma treatment and management are determined by the cell type, location, and grade of malignancy. ${ }^{2}$ Glioma grade set to become a vital factor for treatment decision. ${ }^{3}$ According to the WHO report, glioma are classified as either 'low-grade' (grade I or II) or 'high-grade' (grade III or IV) depending on their development potential and aggressiveness. ${ }^{4}$ The current gold standard for glioma grading is based on tumor morphologic observations and histopathologic findings. However, these approaches have disadvantages and limitations, such as high intra-observer variation, mistakes in tissue sampling during the biopsy, and invasive procedures., ${ }^{5,6}$ Researchers have proposed non-invasive imaging techniques to overcome these limitations.

Thanks to high soft-tissue contrast, non-ionizing radiation, high resolution, and anatomical and functional imaging, Magnetic Resonance Imaging (MRI) has become an essential medical imaging modality in brain imaging. Various MRI imaging protocols have been suggested for glioma grading, such as Susceptibility-weighted imaging (SWI) ${ }^{7}$, Diffusion-weighted imaging (DWI) ${ }^{8}$, Susceptibility contrast enhancement (SCE) ${ }^{9}$, Dynamic contrast enhancement (DCE) ${ }^{\mathbf{1 0}}$, Arterial spin labelling $(\mathrm{ASL})^{11}$, Magnetic resonance spectroscopy (MRS) ${ }^{12}$, etc.

SWI is an MRI technique for detecting and characterizing tissue components based on the difference in tissue susceptibility properties. ${ }^{13} \mathrm{SWI}$ is an essential and accurate MRI imaging protocol to diagnose the disease and presence of paramagnetic substance such as iron, blood products, and hemorrhages. ${ }^{14,15}$ It has now been demonstrated that with increasing glioma grade, micro bleeding and hemorrhages inside the tumor were observed..$^{15,16}$ Because hemorrhage and micro bleeding (specifically blood products) can change the local susceptibility properties, they can easily be detected with SWI. In a major advance in 2014, Ding et al. ${ }^{17}$ demonstrated the SWI capability for glioma grading. They concluded that we could use SWI images for primary central nervous system lymphoma

(c) 2021 Amir Khorasani, Mohamad Bagher Tavakoli, Masih Saboori. This is an open access article licensed under the Creative Commons Attribution-NonCommercialNoDerivs License (http://creativecommons.org/licenses/by-nc-nd/4.0/). 
(PCNSLs) from high-grade glioma and brain metastases differentiation with the area under the curve (AUC) of 0.873 .

DWI is one of the advanced MRI imaging sequences which is based on measuring the random Brownian motion of water molecules of tissue. ${ }^{18}$. The typical DWI protocol produces us DWI images and apparent diffusion coefficient (ADC) maps. ADC maps have become an essential issue in tumor grading with MRI. ${ }^{19}$ Therefore, much work on ADC's potential for glioma grading has been carried out. ${ }^{\text {19-22 }}$

Fluid attenuated inversion recovery (FLAIR) is an anatomical and conventional MRI inversion recovery pulse sequence that removes cerebrospinal fluid (CSF) signals from the images. The contrast of the brain on FLAIR images is similar to $\mathrm{T}_{2}$-weighted images, but CSF is dark. The FLAIR's usefulness in the diagnostic of many central nervous system disorders such as cortical, meningeal, and periventricular diseases has been evaluated.

One of the main issues we know about glioma grading with different MRI protocols is a lack of sufficient diagnostic value of these images. Therefore, there are still some low diagnostic accuracy issues. Much work on the potential of data combination of different imaging protocols for glioma grading has been carried out. ${ }^{23-25}$ However, there are still some critical issues. One way to overcome this limitation would be medical image fusion. Using the medical image fusion techniques, we combine the information of two different images and display them as one image. The result will be an increase in the diagnostic accuracy of the fused image. There are two kinds of image fusion methods: spatial and transform domain approaches. ${ }^{26,27}$ In medical image fusion, transform domain-based methods are commonly used. ${ }^{\mathbf{2 8 , 2 9}}$ Source images are firstly transformed into specific coefficients by transform domain methods. The source image coefficients are then fused, and the fused image is reconstructed by inversely transforming all of the fused coefficients.

Multi-scale transform (MST), as a transform domain-based and frequency domain method, has recently attracted particular attention for medical image fusion tasks. ${ }^{30}$ The MST-based algorithm divided source images into high and low-frequency sub-images using a transform and then generated separate fusion rules for the high and low-frequency sub-images to reconstruct the final fused images. One of the MST-based algorithms used for medical image fusion was Laplacian Re-decomposition (LRD) fusion algorithm. ${ }^{30}$ This paper examines the significance of the LRD medical fusion algorithm in the rise of diagnostic accuracy for glioma grading with the fusion of SWI, ADC, and FLAIR images. In the light of the foregoing, we tried to use SWI images, T2-FLAIR images, and ADC maps in the current study as the input images of the LRD fusion algorithm for glioma grading purposes. The aim of this study is to evaluate the usefulness of the LRD image fusion algorithm for brain tumor grading with SWI, T2-FLAIR, and ADC map and improve the grading accuracy for brain tumors with the fusion of advanced MRI images.

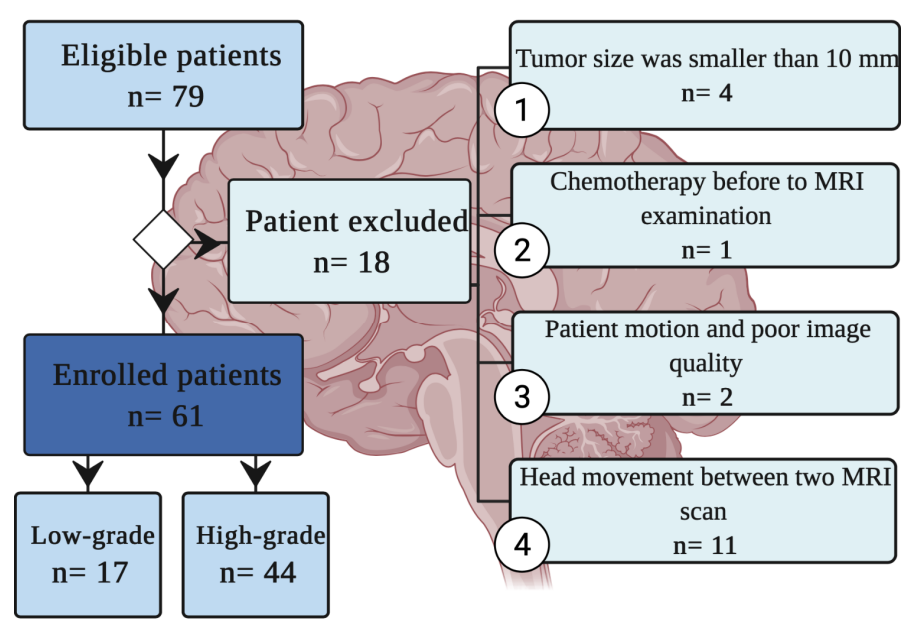

Figure 1. Patient population flow-chart of study.

\section{Material and methods}

\section{Patients}

This study included 79 patients with histologically confirmed glioma by surgical resection who underwent magnetic resonance examination between April 2020 and February 2021. One experienced neuropathologist examined the tumor specimens according to the WHO 2016 classification. ${ }^{4}$

Of these patients, 61 of them ( 32 men, 29 women; mean age 53.16 years; range [23 85] years), 17 low-grade gliomas and 44 high-grade gliomas) were included (Figure 1) under the following criteria in this study: (1) histopathology confirmation of the glioma; (2) no radiotherapy or chemotherapy performed before the magnetic resonance imaging (MRI) examination; (3) lesion equal to or greater than $10 \mathrm{~mm}$; (4) adequate image quality (without patient motion or susceptibility artifact); (5) do not have an allergy to the gadolinium-based contrast agent. So, a total of 61 glioma patients were analyzed in this study. Figure 1 shows a flow chart of the study population. Before the MRI scan, all the patients provided informed consent, and the local research ethics committee has approved the study.

\section{MRI imaging}

MRI imaging examinations were performed at a $1.5 \mathrm{~T}$ scanner (GE MRI Signa Explorer 1.5T) using a 16-channel head coil with the patient in a supine position. The imaging protocols included: a 3-plane localizer sequence, axial T1 pre-contrast $(\mathrm{TR}=400 \mathrm{~ms}, \mathrm{TE}=10 \mathrm{~ms}, \mathrm{FA}=90$, Slice thickness $=5 \mathrm{~mm}$, and spacing $=5 \mathrm{~mm})$ and post-contrast $(\mathrm{TR}=6.1 \mathrm{~ms}$, $\mathrm{TE}=2.2 \mathrm{~ms}, \mathrm{TI}=20 \mathrm{~ms}, \mathrm{FA}=12$, Slice thickness $=5 \mathrm{~mm}$, and spacing $=5 \mathrm{~mm})$, axial echo-planar DWI imaging $(\mathrm{TR}=$ $5268 \mathrm{~ms}, \mathrm{TE}=113.2 \mathrm{~ms}, \mathrm{FA}=90$, Slice thickness $=5 \mathrm{~mm}$, and spacing $=5 \mathrm{~mm}$ with $b$-values $=50,1000)$, axial SWI imaging $(\mathrm{TR}=87 \mathrm{~ms}, \mathrm{TE}=47.5 \mathrm{~ms}, \mathrm{FA}=25$, Slice thickness $=3 \mathrm{~mm}$, and spacing $=1.5 \mathrm{~mm}$, MR Acquisition Type: 3D), and axial T2-FLAIR (T2F) weighted $(\mathrm{TR}=8500 \mathrm{~ms}, \mathrm{TE}=97.05 \mathrm{~ms}$, $\mathrm{TI}=2503 \mathrm{~ms}, \quad \mathrm{FA}=170, \quad$ Slice thickness $=5 \mathrm{~mm}, \quad$ and 
spacing $=5 \mathrm{~mm}$ ) imaging. DWI and SWI imaging protocols were acquired before the administration of contrast media.

Raising the starting b-value to 50 , instead of 0 , provided a clear visualization of the tumor image and decreased T2 shine through artifact. DWI MRI data were collected in three orthogonal directions and combined to produce a trace image. By using these data, ADC maps were created on a pixel-by-pixel basis with an MRI imaging unit workstation.

Using quadrature detection, both magnitude and phase data are separately reconstructed to magnitude and phase images. First, the magnitude image is saved. Then, a phase mask is next created that scales data from the filtered phase images over a $0-1$ range to accentuate tissues with different SWI image.

\section{Image fusion}

For the image fusion process, the patient images were transferred from the picture archiving and communication system (PACS) to a personal computer (PC) (Figure 2) and resized to $256 \times 256$. Through the image resize, we were able to reduce the fusion time and code run time. The fusion algorithm used in this study is practically the same as the one proposed by Li et al. ${ }^{30}$ using MATLAB 2019a Mathworks. We selected the LRD medical image fusion algorithm due to its better performance than the other medical image fusion methods. ${ }^{\text {30-32 }}$

Briefly, the Laplacian Re-decomposition (LRD) medical fusion algorithm process (Figure 3) started with Gradient Domain Image Enhancement (GDIE) component. GDIE includes four steps: Maximum Local Difference (MLD), interval division of feature information, remapping gradient feature, and convolution operation. The result of the GDIE function on each source image is an enhancement image $\left(\mathrm{H}_{\mathrm{A}}\right.$ and $\left.\mathrm{H}_{\mathrm{B}}\right)$ that highlight the edge and detail information of source images. In the next stage of the LRD process, Laplacian Pyramid (LP) transform decomposes the $\mathrm{H}_{\mathrm{A}}$ and $\mathrm{H}_{\mathrm{B}}$ into High-frequency subband images $\left(\mathrm{L}_{\mathrm{A}}\right.$ and $\left.\mathrm{L}_{\mathrm{B}}\right)$ with edge and details information and Low-frequency sub-band images $\left(\mathrm{G}_{\mathrm{A}}\right.$ and $\left.\mathrm{G}_{\mathrm{B}}\right)$ with background information. In the third stage of the LRD medical fusion algorithm process, Decision Graph Re-decomposition (DGR) was used. The DGR could properly classify the complementary and redundant data of $\mathrm{L}_{\mathrm{A}}$ and $\mathrm{L}_{\mathrm{B}}$ by designing two classifiers. The first classifier could efficiently separate redundant data of $\mathrm{L}_{\mathrm{A}}$ and $\mathrm{L}_{\mathrm{B}}$ into overlapping domain images $\left(\mathrm{O}_{\mathrm{A}}\right.$ and $\left.\mathrm{O}_{\mathrm{B}}\right)$. The second classifier of the DGR function stores complementary information of $\mathrm{L}_{\mathrm{A}}$ and $\mathrm{L}_{\mathrm{B}}$ in the image of the Non-overlapping domain $\left(\mathrm{N}_{\mathrm{A}}\right.$ and $\left.\mathrm{N}_{\mathrm{B}}\right)$. In the next step of the LRD medical fusion algorithm, we fuse the images created from the previous steps by several fusion rules. $G_{A}$ and $G_{B}$ with Local Energy Maximum (LEM) fusion rule were fused to form Low-frequency sub-band fusion image $\left(\mathrm{G}_{\mathrm{F}}\right)$.

To create the overlapping domain fusion image $\left(\mathrm{O}_{\mathrm{F}}\right)$ containing both source images' feature images, the overlapping domain (OD) fusion rule was used. According to the definition of the Non-overlapping domain by DGR, the fusion image $\left(\mathrm{N}_{\mathrm{F}}\right)$ of $\mathrm{N}_{\mathrm{A}}$ and $\mathrm{N}_{\mathrm{B}}$ could be obtained by the Non-overlapping domain (NOD) fusion rule. To reconstruct the High-frequency sub-band fusion image $\left(\mathrm{L}_{\mathrm{F}}\right)$ by fusing $\mathrm{O}_{\mathrm{F}}$ and $\mathrm{N}_{\mathrm{F}}$ and eliminating image artifacts, Inverse Re-decomposition Scheme (IRS) fusion rule was proposed. Finally, the inverse LP method reconstructs the final fused image $(F)$ from $G_{F}$ and $L_{F}$ images. More details of this medical fusion algorithm are in ${ }^{\mathbf{3 0}}$.

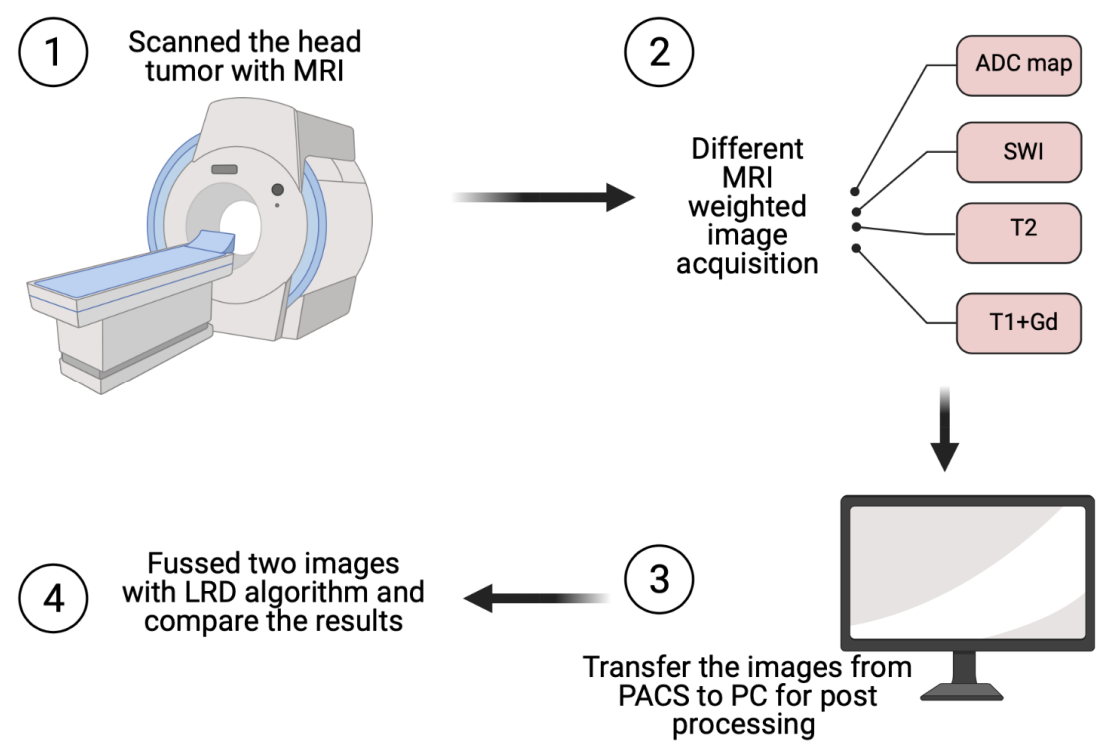

Figure 2. Workflow of the study - methodology abstract 


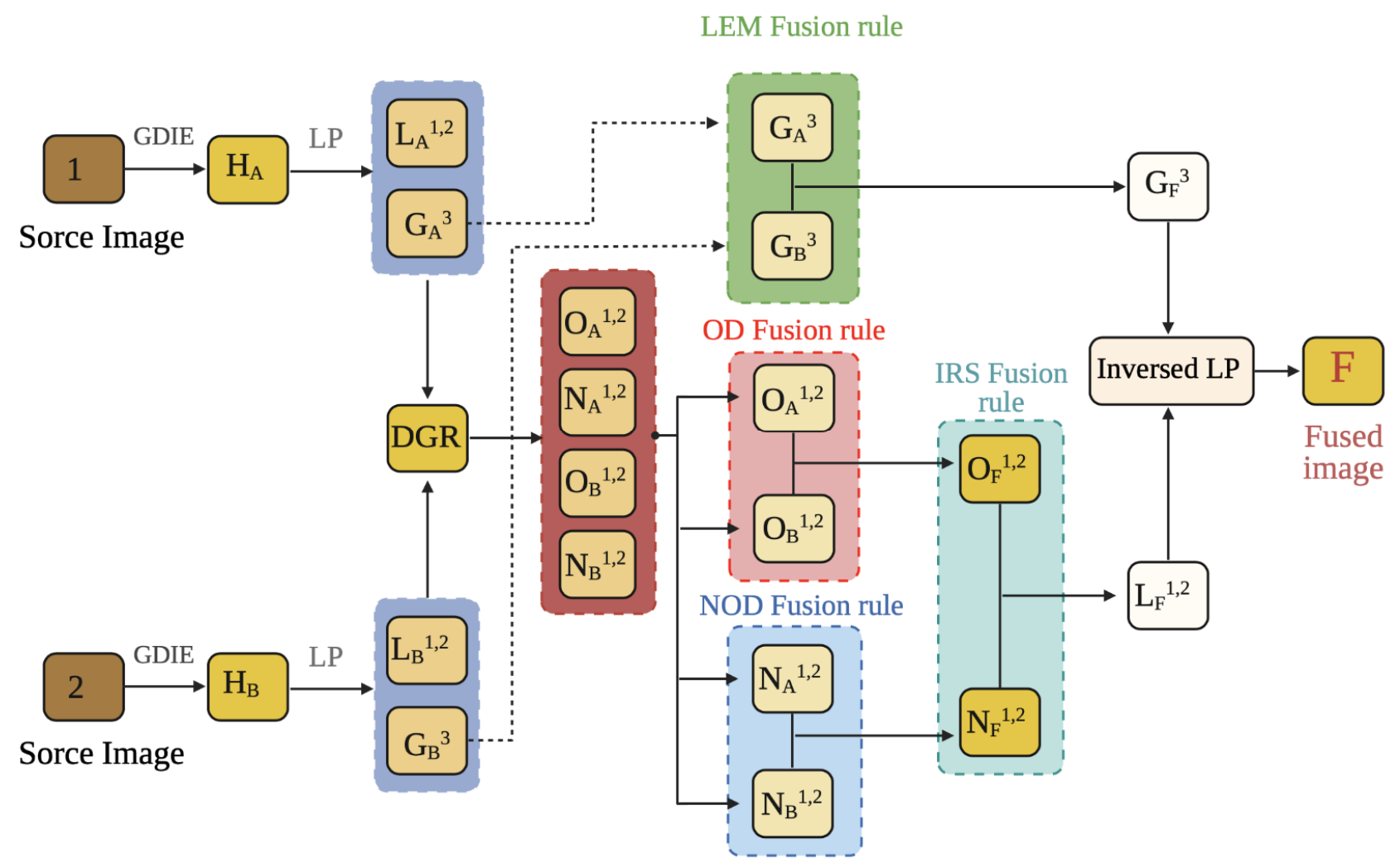

Figure 3. Laplacian Re-decomposition (LRD) algorithm for medical image fusion includes several steps. HA and HB: enhancement images, LA and LB: High-frequency sub-band images, GA and GB: Low-frequency sub-band images, OA and OB: overlapping domain images, NA and NB: Non-overlapping domain images, OF: overlapping domain fusion images, NF: Non-overlapping domain fusion images, GF: lowfrequency sub-band fusion images, LF: high-frequency sub-band fusion images.

\section{Data evaluation}

For each patient, a slice with the largest tumor size was selected and analyzed. Two circular regions of interest (ROI) for each patient were manually drawn by a radiologist in the T1 images with gadolinium injection (T1Gd) with the ImageJ software. These two ROIs are located: (a) in the normal white matter (WM) as the background value for normalization process, and (b) in the enhancement region (ER) on T1Gd images for highgrade glioma and high-signal intensity region on T2F-weighted images for low-grade glioma because on low-grade tumors the enhancement in T1Gd is scarce. ${ }^{33}$ The ROIs drawn in the T1Gd and $\mathrm{T} 2 \mathrm{~F}$ images were transferred to the co-aligned SWI image and ADC maps and fused images (SWI+T2F, SWI+ADC, and $\mathrm{T} 2 \mathrm{~F}+\mathrm{ADC}$ ) to calculate the signal intensity of each ROI. Three ROIs were sampled and averaged for each region.

The Relative Signal Contrast (RSC) was calculated by:

$R S C_{R O I}=\frac{\mu_{R O I}-\mu_{W M}}{\mu_{W M}}$

Eq. 1

Where, $\mu_{R O I}$ is the mean signal intensity of the ROI, and $\mu_{W M}$ is the mean signal intensity of the normal white matter as the mean background values in each picture. Six RSC were calculated, $R S C_{S W I}, \quad R S C_{A D C}, \quad R S C_{T 2 F}, \quad R S C_{S W I+T 2 F}, \quad R S C_{S W I+A D C}$, $R S C_{T 2 F+A D C}$, and evaluated for brain tumor grading.

\section{Statistical analysis}

Statistical analyses were carried out using SPSS 26.0 software (IBM Corp. Armonk, NY, USA). The two-tailed unpaired student t-test was performed to statistically analyze the mean signal value and RSCs between low and high-grade glioma. Moreover, the Exact Fisher test was used for the assessment of the sex and age relationship with the tumor grade. RSCs values were presented as the mean \pm standard deviation. The area under the curve (AUC), sensitivity, and specificity of the receiver operating characteristic (ROC) curve for the significant parameters was calculated. The cut-off value of significant parameters in ROC analysis was calculated by setting the maximum Youden index. $\mathrm{P}<0.05$ was indicated to be statistically significant. 


\section{Results}

The study consisted of 17 low-grade (27.86\%) and 44 highgrade $(72.14 \%)$ glioma. The histopathologic findings are presented in Table 1. There was no significant difference in gender and age between low-grade and high-grade glioma (Table 1)

Table 1. Characteristics of patients with low-grade and high-grade gliomas.

\begin{tabular}{ccccc}
\hline \hline & Low-grade & High-grade & P-value \\
\hline \multicolumn{2}{c}{ Mean age (years) } & $49.18 \pm 15.17$ & $56.89 \pm 16.33$ & 0.25 \\
\multirow{2}{*}{ Sex } & Man & 8 & 24 & 0.99 \\
& Woman & 9 & 20 & \\
\hline \hline
\end{tabular}

No significant difference was observed in mean signal intensity values of normal-appearing white matter (WM) between the low-grade and high-grade glioma in all MRI imaging protocols of the current study (P-value > 0.05). In Figure 4, you can see some of the results of the LRD fusion algorithm on a patient with high-grade glioma.

The average RSCs with standard deviation for low-grade and high-grade glioma in differently weighted images were shown in Figure 5. No significant difference was detected in $R S C_{T 2 F}$ and $R S C_{T 2 F+A D C}$ between low and high-grade glioma. Statistical tests revealed that the mean $R S C_{S W I}$, and $R S C_{A D C}$ and some fused images $\left(R S C_{S W I+T 2 F}, R S C_{S W I+A D C}\right)$ were significantly different between low and high-grade glioma (Figure 5). Interestingly, for high-grade glioma, in comparison with lowgrade glioma, lower values of $R S C_{S W I}, R S C_{A D C}, R S C_{S W I+T 2 F}$, and $R S C_{S W I+A D C}$ were found.

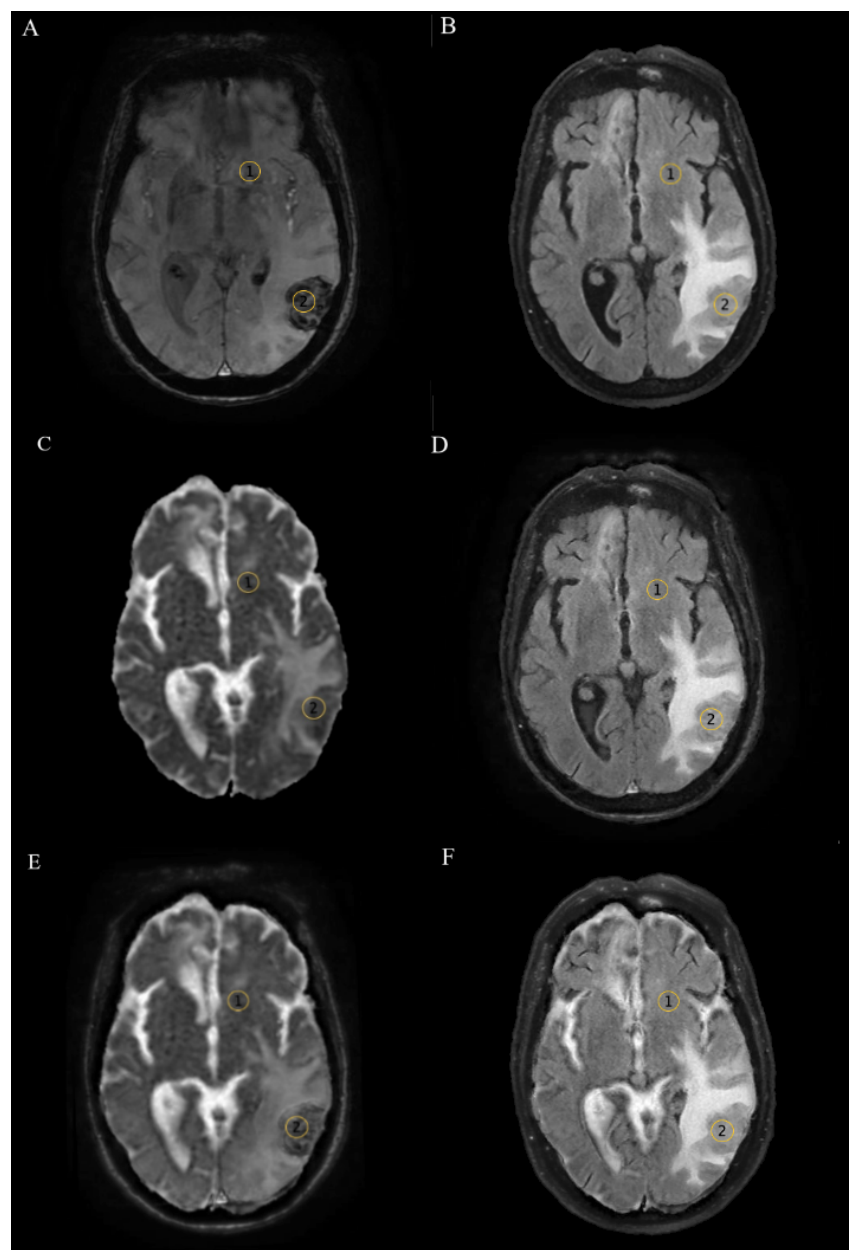

Figure 4. The image of a 71-year-old woman with high-grade glioma. (A) axial SWI (B) axial T2-FLAIR (C) axial ADC map (D) fused image of SWI+T2F with LRD fusion algorithm. (E) fused image of SWI+ADC with LRD fusion algorithm. (F) fused image of T2F+ADC with LRD fusion algorithm. In all images, ROI 1 is the white matter region (WM), and ROI 2 is the enhancement region on T1Gd.

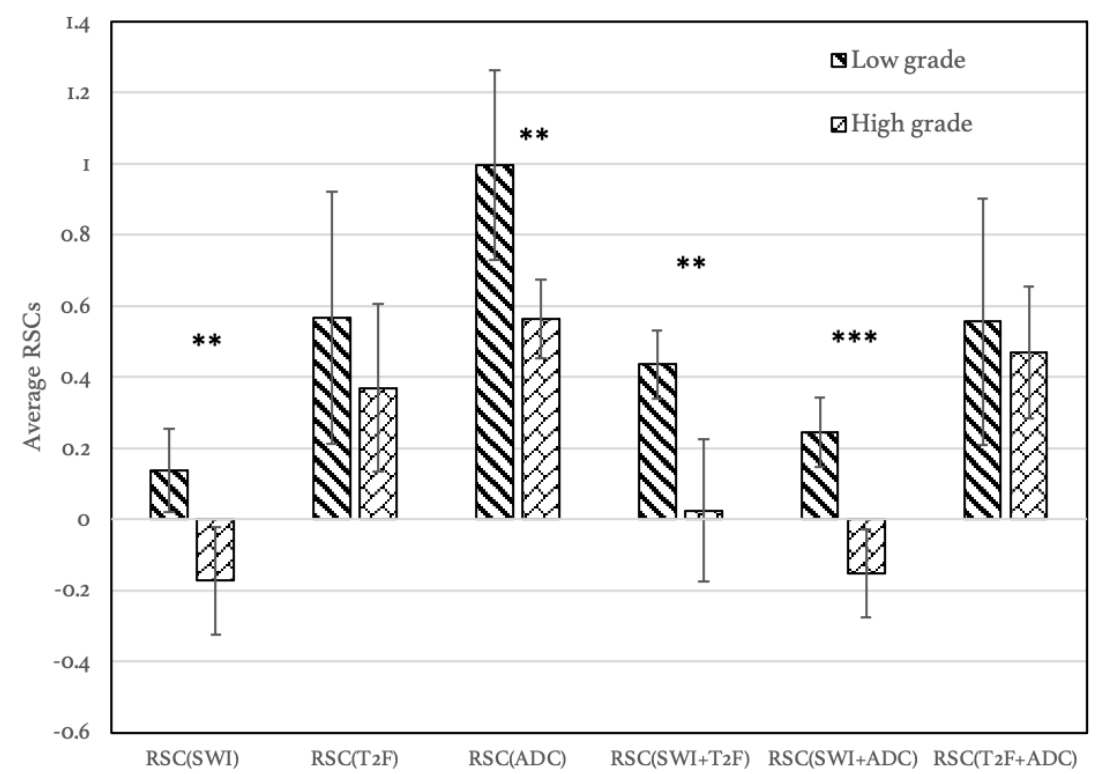

Figure 5. Average RSCs in SWI, T2F, ADC map, and fused images (SWI+T2F, SWI+ADC, and R2F+ADC) of low and high-grade glioma (* P-value $<0.05 ; * * \mathbf{P}$-value $<0.01 ; * *$ P-value $<0.001$ ) 
Table 2. ROC parameters in SWI, ADC map, and fused image (SWI+T2F, and SWI+ADC) for differentiation of low and high-grade glioma.

\begin{tabular}{|c|c|c|c|c|c|}
\hline & RSC Cutoff value & AUC & Sensitivity & Specificity & Maximum Youden Index \\
\hline$R S C_{S W I}$ & 0.062 & 0.871 & 0.814 & 1 & 0.814 \\
\hline$R S C_{A D C}$ & 0.726 & 0.833 & 0.674 & 0.842 & 0.516 \\
\hline$R S C_{S W I+T 2 F}$ & 0.303 & 0.860 & 0.872 & 0.833 & 0.705 \\
\hline$R S C_{S W I+A D C}$ & 0.168 & 0.978 & 0.916 & 1 & 0.916 \\
\hline
\end{tabular}

To distinguish between low and high-grade glioma in different MRI imaging protocols and fused images and assess the diagnostic value of these images, ROC curve analysis was used (Figure 6). Table 2 represents the cut-off value, an area under curve (AUC), sensitivity, specificity, and maximum Youden index for RSCs in SWI, ADC, and fused images (SWI+T2F, and $\mathrm{SWI}+\mathrm{ADC}$ ) for discrimination of low and high-grade glioma. The analysis did not reveal any significant difference in $R S C_{T 2 \mathrm{~F}}$ and $R S C_{T 2 \mathrm{~F}+A D C}$ between low and high-grade glioma. So further analysis on the ROC curve of these images was not performed.

The most remarkable result to emerge from Table $\mathbf{2}$ is that the AUC for low and high-grade glioma differentiation, with RCSs cutoff-value 0.062 and 0.726 , was 0.871 and 0.833 in SWI and ADC map, respectively. Image fusion of SWI and ADC map with $R S C_{S W I+A D C}$ cut-off value of 0.168 increased the AUC to 0.978 (12.3\% improvement toward SWI images and $17.4 \%$ improvement toward ADC map) (Table 2) to low and highgrade glioma differentiation by LRD medical image fusion algorithm.

\section{Discussion}

Accurate glioma grading became a vital factor in glioma treatment management and treatment decision. Despite its shortcoming, invasiveness, and time-consuming, biopsies and sampling have been widely used as the gold standard for glioma grading in the clinic. The current solution to overcome these limitations is using advanced and functional imaging modality such as MRI. Through the use of MRI as a medical imaging modality for brain imaging, we were able to reconstruct and acquire images with high soft-tissue contrast, non-invasive process, non-ionizing imaging modality, anatomical and functional imaging. Recent development in MRI imaging protocols has led to the use of this modality for glioma grading. One of the main issues in our knowledge of glioma grading with different MRI imaging protocols is a lack of proper diagnostic value of these images. In the light of recent development in medical image fusion methods and fusing the different images, there is now a considerable solution to overcome these limitations for glioma grading with MRI.

The analysis did not confirm any significant difference in $R S C_{T 2 F}$ between low and high-grade glioma. There are several possible explanations for this result. A possible explanation may be that conventional MRI imaging protocols, such as T1 and T2F-weighted images, provide valuable structural information about glioma dominated by tissue water content but limited

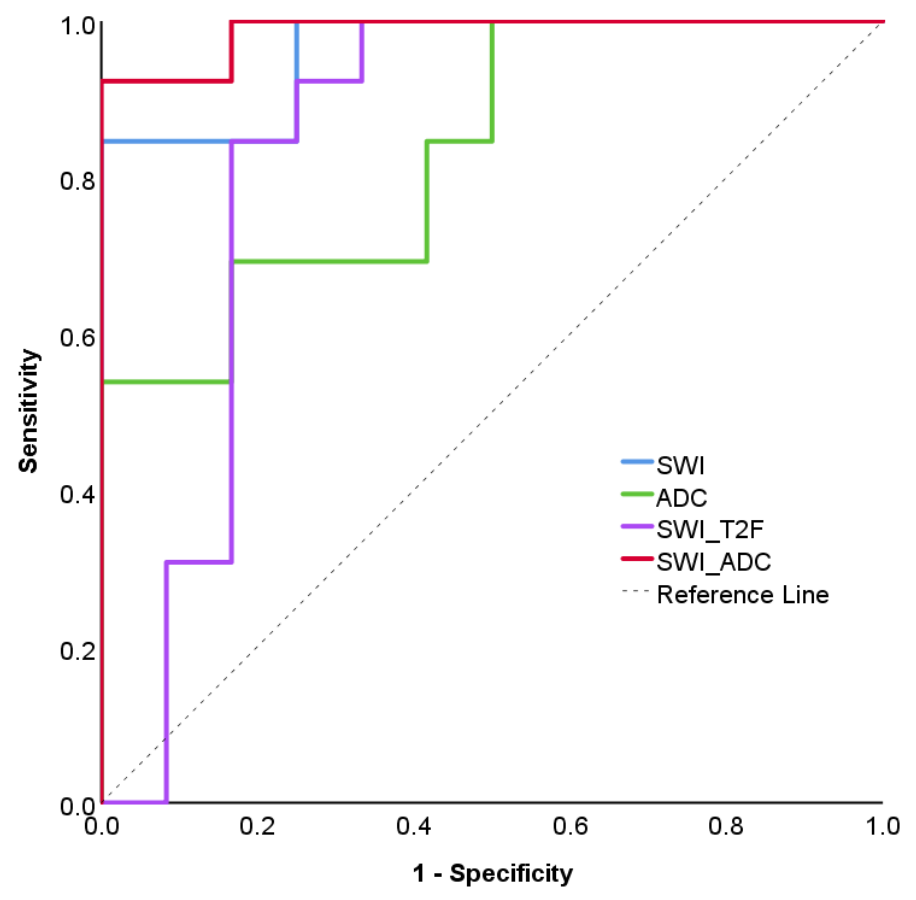

Figure 6. ROC curve of SWI, ADC, and fused images (SWI+T2F, and SWI+ADC) for differentiation of low-grade and high-grade glioma.

physiological details. This structural information is not enough for glioma grading. As suggested by previous publications, ${ }^{34}$ the evidence we found points to the low diagnostic value of conventional T2 images for low and high-grade glioma differentiation.

Significantly, $R S C_{S W I}$, and $R S C_{A D C}$ and some fused images $\left(R S C_{S W I+T 2 F}, R S C_{S W I+A D C}\right)$ were different between low and high-grade glioma. According to our results, it is interesting to note that the $R S C_{S W I}, R S C_{A D C}, R S C_{S W I+T 2 F}$, and $R S C_{S W I+A D C}$ decreased significantly with glioma grade increased.

We believed that the relationship between $R S C_{A D C}$ and glioma grade is related to tumor cellularity. An increasing number of studies have found that the tumor cellularity was decreased with tumor grade. ${ }^{19-22}$ ADC map provides information about the water molecules' motility and diffusion coefficient in the tissue. With cellularity increase in the tissues, motility of water molecules was restricted, and ADC value was decreased. ${ }^{20,21}$ So, there was a significant negative correlation between mean $R S C_{A D C}$ values and tumor grade. This is in complete agreement with previous results. ${ }^{19,20,35-37}$ 
Significantly, $R S C_{S W I}$ are decreased with glioma grade increase (Figure 5). SWI imaging protocol is very sensitive and advanced MRI imaging. Paramagnetic substances such as iron and blood product appear dark and have low signal intensity on SWI images. The reason is that the presence of these substances changes the susceptibility properties of the tissue. Several studies, for example ${ }^{38}$, and ${ }^{39}$, have highlighted that as the tumor grade increases, the micro-bleeding and vascularization inside the tumor also increase. Our study provides additional support for $R S C_{S W I}$ decrease with glioma grade increase because of the presence of micro-bleeding and tumor vascularization in highgrade glioma. These values correlate satisfactorily well with Wasif et al. ${ }^{40}$ and further support the role of micro-bleeding on image darkness in SWI images.

We used LRD medical fusion algorithm to fuse two different MRI images and increase the diagnostic value of the fused image for glioma grading. It was found that AUC, sensitivity, and specificity for low and high-grade glioma differentiation in SWI and ADC map fusion (SWI+ADC) was significantly higher than other images. This finding points to the usefulness of the LRD fusion algorithm as a method for increasing the diagnostic value of the different images by combining the source image data. The most striking result to emerge from Table 2 and Figure 6 is that the fused image of ADC map and SWI (SWI+ADC) has a higher diagnostic value for glioma grading than the individual SWI and ADC images. It is important to note that, the ADC map contrast is based on water molecules motility, and SWI contrast is based on susceptibility properties of tissue. By fusing ADC map and SWI images using the LRD medical fusion algorithm, a final image will be reconstructed containing the data of ADC map (water molecules motility) and SWI (susceptibility properties) images simultaneously. Remarkably, some fused images which were fused with T2F images, such as SWI+T2F and ADC+T2F have lower AUC, sensitivity, and specificity than SWI+ADC fused images. In our view, this relationship is related to statistical meaningless $R S C_{T 2 F}$ difference between low and high-grade glioma. We are aware that our research may have limitations. Because the aim of this study is to examine the LRD medical image fusion algorithm's usefulness for glioma grading with SWI, ADC map, and T2F, small sample size was chosen.

\section{Conclusion}

This paper has investigated the use of the LRD medical fusion algorithm for glioma grading with SWI, ADC map, and T2F MRI images. The most important limitation is due to the small sample size of the low-grade group. Future work should focus on larger sample size and population. The evidence from this study points towards the idea that, with the LRD medical fusion algorithm, we were able to improve the diagnostic values of MRI images for glioma grading. The findings of this study suggest that by fusing ADC map and SWI images, the highest AUC, sensitivity, and specificity to low and high-grade differentiation can be achieved. The present findings have important implications for an alternative method of biopsy and surgical resection for glioma grading. Future work will investigate fusing different MRI images weights and protocol for glioma grading by the LRD medical fusion algorithm.

\section{Conflicts of Interest}

The authors declare that they have no conflict of interests,

\section{Funding Statement}

This study was supported by Isfahan University of Medical Sciences, Isfahan, I.R. Iran (grant number 399077).

\section{Acknowledgment}

The authors thank Isfahan University of Medical Sciences for the financial support of this work.

\section{List of abbreviations}

LRD Laplacian Re-decomposition MRI magnetic resonance imaging

T1Gd T1 post enhancement

ADC apparent diffusion coefficient

ROC receiver operating characteristic curve

RSC Relative Signal Contrast

AUC Aera Under Curve

SWI Susceptibility-weighted imaging

DWI Diffusion-weighted imaging

SCE Susceptibility contrast enhancement

DCE Dynamic contrast enhancement

MRS Magnetic resonance spectroscopy

MST Multi-scale transform

TR repetition time

TE time of echo

TI time of inversion

FA flip angle

PACS picture archiving and communication system

GDIE Gradient Domain Image Enhancement

MLD Maximum Local Difference

LP Laplacian Pyramid

DGR Decision Graph Re-decomposition

LEM Local Energy Maximum

OD overlapping domain

NOD Non-overlapping domain

IRS Inverse Re-decomposition Scheme

ROI regions of interest

FLAIR Fluid attenuated inversion recovery 


\section{References}

1. Goodenberger ML, Jenkins RB. Genetics of adult glioma. Cancer Genet. 2012;205(12):613-621. https://doi.org/10.1016/j.cancergen.2012.10.009

2. Sasaki S, Tomomasa R, Nobusawa S, et al. Anaplastic pleomorphic xanthoastrocytoma associated with an H3G34 mutation: a case report with review of literature. Brain Tumor Pathol. 2019;36(4):169-173. https://doi.org/10.1007/s10014-019-00349-8

3. Hakyemez B, Erdogan C, Ercan I, Ergin N, Uysal S, Atahan S. High-grade and low-grade gliomas: differentiation by using perfusion MR imaging. Clin Radiol. 2005;60(4):493-502. https://doi.org/10.1016/j.crad.2004.09.009

4. Louis DN, Perry A, Reifenberger G, et al. The 2016 World Health Organization classification of tumors of the central nervous system: a summary. Acta Neuropathol. 2016;131(6):803-820. https://doi.org/10.1007/s00401-016-1545-1

5. Law M, Oh S, Babb JS. Low-grade gliomas: Dynamic susceptibility-weighted contrast-enhanced perfusion MR imaging-prediction of patient clinical rewsponse (Radiology (2006) 238,(658-667)). Radiology. 2008;246(3):989. https://doi.org/10.1148/radiol.2382042180

6. Arvinda HR, Kesavadas C, Sarma PS, et al. RETRACTED ARTICLE: Glioma grading: sensitivity, specificity, positive and negative predictive values of diffusion and perfusion imaging. J Neurooncol. 2009;94(1):87-96. https://doi.org/10.1007/s11060-009-9807-6

7. Hsu CC, Watkins TW, Kwan GNC, Haacke EM. Susceptibility-weighted imaging of glioma: update on current imaging status and future directions. J Neuroimaging. 2016;26(4):383-390. https://doi.org/10.1111/jon.12360

8. Ryu YJ, Choi SH, Park SJ, Yun TJ, Kim J-H, Sohn C-H. Glioma: application of whole-tumor texture analysis of diffusion-weighted imaging for the evaluation of tumor heterogeneity. PLoS One. 2014;9(9):e108335. https://doi.org/10.1371/journal.pone.0108335

9. Santarosa C, Castellano A, Conte GM, et al. Dynamic contrast-enhanced and dynamic susceptibility contrast perfusion MR imaging for glioma grading: preliminary comparison of vessel compartment and permeability parameters using hotspot and histogram analysis. Eur J Radiol. 2016;85(6):1147-1156. https://doi.org/10.1016/j.ejrad.2016.03.020

10. Jain KK, Sahoo P, Tyagi R, et al. Prospective glioma grading using single-dose dynamic contrast-enhanced perfusion MRI. Clin Radiol. 2015;70(10):1128-1135. https://doi.org/10.1016/j.crad.2015.06.076

11. Kim HS, Kim SY. A prospective study on the added value of pulsed arterial spin-labeling and apparent diffusion coefficients in the grading of gliomas. Am J Neuroradiol. 2007;28(9):1693-1699. https://doi.org/10.3174/ajnr.A0674

12. Wang Q, Zhang H, Zhang J, et al. The diagnostic performance of magnetic resonance spectroscopy in differentiating high-from lowgrade gliomas: a systematic review and meta-analysis. Eur Radiol. 2016;26(8):2670-2684. https://doi.org/10.1007/s00330-015-4046-z

13. Schenck JF. The role of magnetic susceptibility in magnetic resonance imaging: MRI magnetic compatibility of the first and second kinds. Med Phys. 1996;23(6):815-850. https://doi.org/10.1118/1.597854

14. Mittal S, Wu Z, Neelavalli J, Haacke EM. Susceptibility-weighted imaging: technical aspects and clinical applications, part 2. Am J Neuroradiol. 2009;30(2):232-252. https://doi.org/10.3174/ajnr.A1461

15. Sehgal V, Delproposto Z, Haddar D, et al. Susceptibility-weighted imaging to visualize blood products and improve tumor contrast in the study of brain masses. J Magn Reson Imaging An Off J Int Soc Magn Reson Med. 2006;24(1):41-51. https://doi.org/10.1002/jmri.20598

16. Li C, Ai B, Li Y, Qi H, Wu L. Susceptibility-weighted imaging in grading brain astrocytomas. Eur J Radiol. 2010;75(1):e81-e85. https://doi.org/10.1016/j.ejrad.2009.08.003

17. Ding Y, Xing Z, Liu B, Lin X, Cao D. Differentiation of primary central nervous system lymphoma from high-grade glioma and brain metastases using susceptibility-weighted imaging. Brain Behav. 2014;4(6):841-849. https://doi.org/10.1002/brb3.288

18. Minati L, Węglarz WP. Physical foundations, models, and methods of diffusion magnetic resonance imaging of the brain: A review. Concepts Magn Reson Part A An Educ J. 2007;30(5):278-307. https://doi.org/10.1002/cmr.a.20094

19. Wang Q, Lei D, Yuan Y, Xiong N. Accuracy of ADC derived from DWI for differentiating high-grade from low-grade gliomas: Systematic review and meta-analysis. Medicine (Baltimore). 2020;99(8). https://doi.org/10.1097/MD.0000000000019254

20. Soliman RK, Essa AA, Elhakeem AAS, Gamal SA, Zaitoun MMA. Texture analysis of apparent diffusion coefficient (ADC) map for glioma grading: Analysis of whole tumoral and peri-tumoral tissue. Diagn Interv Imaging. 2021;102(5):287-295. https://doi.org/10.1016/j.diii.2020.12.001

21. Phuttharak W, Thammaroj J, Wara-Asawapati S, Panpeng K. Grading Gliomas Capability: Comparison between Visual Assessment and Apparent Diffusion Coefficient (ADC) Value Measurement on Diffusion-Weighted Imaging (DWI). Asian Pacific J Cancer Prev APJCP. 2020;21(2):385. https://doi.org/10.31557/APJCP.2020.21.2.385

22. Sadeghi N, D'haene N, Decaestecker C, et al. Apparent diffusion coefficient and cerebral blood volume in brain gliomas: relation to tumor cell density and tumor microvessel density based on stereotactic biopsies. Am J Neuroradiol. 2008;29(3):476-482. https://doi.org/10.3174/ajnr.A0851

23. Ma X, Lv K, Sheng J, et al. Application evaluation of DCE-MRI combined with quantitative analysis of DWI for the diagnosis of prostate cancer. Oncol Lett. 2019;17(3):3077-3084. https://doi.org/10.3892/ol.2019.9988 
24. Hilario A, Ramos A, Perez-Nunez A, et al. The added value of apparent diffusion coefficient to cerebral blood volume in the preoperative grading of diffuse gliomas. Am J Neuroradiol. 2012;33(4):701-707. https://doi.org/10.3174/ajnr.A2846

25. Saini J, Gupta PK, Sahoo P, et al. Differentiation of grade II/III and grade IV glioma by combining "T1 contrast-enhanced brain perfusion imaging" and susceptibility-weighted quantitative imaging. Neuroradiology. 2018;60(1):43-50. https://doi.org/10.1007/s00234-017-1942-8

26. Qi G, Wang J, Zhang Q, Zeng F, Zhu Z. An integrated dictionary-learning entropy-based medical image fusion framework. Futur Internet. 2017;9(4):61. https://doi.org/10.3390/fi9040061

27. Wang K, Qi G, Zhu Z, Chai Y. A novel geometric dictionary construction approach for sparse representation based image fusion. Entropy. 2017;19(7):306. https://doi.org/10.3390/e19070306

28. Zhu Z, Chai Y, Yin H, Li Y, Liu Z. A novel dictionary learning approach for multi-modality medical image fusion. Neurocomputing. 2016;214:471-482. https://doi.org/10.1016/j.neucom.2016.06.036

29. Zhu Z, Yin H, Chai Y, Li Y, Qi G. A novel multi-modality image fusion method based on image decomposition and sparse representation. Inf Sci (Ny). 2018;432:516-529. https://doi.org/10.1016/j.ins.2017.09.010

30. Li X, Guo X, Han P, Wang X, Li H, Luo T. Laplacian redecomposition for multimodal medical image fusion. IEEE Trans Instrum Meas. 2020;69(9):6880-6890. https://doi.org/10.1109/TIM.2020.2975405

31. Das M, Gupta D, Radeva P, Bakde AM. NSST domain CT-MR neurological image fusion using optimised biologically inspired neural network. IET Image Process. 2020;14(16):4291-4305. https://doi.org/10.1049/iet-ipr.2020.0219

32. Wang G, Li W, Huang Y. Medical image fusion based on hybrid three-layer decomposition model and nuclear norm. Comput Biol Med. 2021;129:104179. https://doi.org/10.1016/j.compbiomed.2020.104179

33. Pouratian N, Asthagiri A, Jagannathan J, Shaffrey ME, Schiff D. Surgery Insight: the role of surgery in the management of low-grade gliomas. Nat Clin Pract Neurol. 2007;3(11):628-639. https://doi.org/10.1038/ncpneuro0634

34. Upadhyay N, Waldman A. Conventional MRI evaluation of gliomas. Br J Radiol. 2011;84(special_issue_2):S107-S111. https://doi.org/10.1259/bjr/65711810

35. Al-Agha M, Abushab K, Quffa K, Al-Agha S, Alajerami Y, Tabash M. Efficiency of High and Standard b Value Diffusion-Weighted Magnetic Resonance Imaging in Grading of Gliomas. J Oncol. 2020;2020. https://doi.org/10.1155/2020/6942406

36. Zhang L, Min Z, Tang M, Chen S, Lei X, Zhang X. The utility of diffusion MRI with quantitative ADC measurements for differentiating high-grade from low-grade cerebral gliomas: evidence from a meta-analysis. J Neurol Sci. 2017;373:9-15. https://doi.org/10.1016/j.jns.2016.12.008

37. Thust SC, Hassanein S, Bisdas S, et al. Apparent diffusion coefficient for molecular subtyping of non-gadolinium-enhancing WHO grade II/III glioma: volumetric segmentation versus two-dimensional region of interest analysis. Eur Radiol. 2018;28(9):3779-3788. https://doi.org/10.1007/s00330-018-5351-0

38. Li X, Zhu Y, Kang H, et al. Glioma grading by microvascular permeability parameters derived from dynamic contrast-enhanced MRI and intratumoral susceptibility signal on susceptibility weighted imaging. Cancer Imaging. 2015;15(1):1-9. https://doi.org/10.1186/s40644-015-0039-z

39. Gaudino S, Marziali G, Pezzullo G, et al. Role of susceptibility-weighted imaging and intratumoral susceptibility signals in grading and differentiating pediatric brain tumors at $1.5 \mathrm{~T}$ : a preliminary study. Neuroradiology. 2020;62(6):705-713. https://doi.org/10.1007/s00234-020-02386-z

40. Mohammed W, Xunning H, Haibin S, Jingzhi M. Clinical applications of susceptibility-weighted imaging in detecting and grading intracranial gliomas: a review. Cancer Imaging. 2013;13(2):186. https://doi.org/10.1102/1470-7330.2013.0020 